\title{
Transient motion due to a moving dislocation in a general anisotropic solid
}

\author{
K.-C. Wu, Taipei, Taiwan \\ (Received September 10, 2001; revised November 7, 2001)
}

\begin{abstract}
Summary. The elastic fields of a dislocation with general Burgers vector starting from rest and moving with constant velocity in a general anisotropic solid are given in closed form. The analysis is made using a sextic formalism that does not require integral transforms. An explicit expression for the energy flux radiated from the dislocation is derived. The drag force on the dislocation is calculated for a class of cubic materials.
\end{abstract}

\section{Introduction}

The problems of moving dislocations that have been studied can be classified into three categories: (i) steady motion of a dislocation moving with constant velocity for all time; (ii) transient motion of a dislocation starting from rest and moving with constant velocity; (iii) transient motion starting from rest and moving with time-dependent velocity. The solution to the first problem is essentially complete for both the isotropic and anisotropic cases as reviewed in [1] and [2]. The second and the third problems have been studied for screw and edge dislocations in isotropic materials ([3]-[5]) and in anisotropic materials ([6]-[8]). In the latter works the dislocation problem was treated as an equivalent half-space problem with mixed boundary conditions. The treatment is strictly valid only for materials with certain symmetries. In the present paper the closed form solution to the second problem is provided for genuine general anisotropic materials and for general types of dislocations.

The customary approach to the analysis of the transient motion is based on integral transform methods and inversion by the Cagniard-de Hoop [9] technique. Recently a sextic formalism for self-similar dynamic problems was proposed by $\mathrm{Wu}$ [10]. In the proposed formalism the general solution is directly expressed in terms of the eigenvalues and eigenvectors of a sixdimensional eigenvalue problem. A major advantage of the proposed formulation is that no integral transforms are required. This fact greatly facilitates derivations of explicit solutions. Here the sextic formalism is employed to treat the moving dislocation problem.

The problem of interest may be considered as the superposition of the following three problems: (i) a static dislocation with the Burgers vector $\boldsymbol{\beta}$ at the origin, (ii) a stationary dislocation with Burgers vector $-\boldsymbol{\beta}$ which suddenly appears at $t=0$ at the origin, and (iii) a moving dislocation with Burgers vector $\boldsymbol{\beta}$ that suddenly appears at $t=0$ at the origin and moving thereafter with constant velocity $\nu$. The solution to the first problem is well known (see [11] for example) and is in fact the solution to the second problem as $t \rightarrow \infty$. The second problem, in turn, is a special case of the third problem with $\nu=0$. Thus one only needs to solve the third problem to construct the desired solution. 
An important quantity in dislocation dynamics is the drag force on the moving dislocation. The drag force is the driving force required for the elastic fields associated with the moving dislocation to have neither an energy source nor an energy sink at the dislocation. Eshelby [12] has given estimates on the drag force due to continuum isotropic elasticity for nonuniformly moving screw dislocations at velocities that are small compared with the shear wave speed. Clifton and Markenscoff [13] have derived the drag force for screw and edge dislocations that start from rest and move thereafter at constant subsonic velocity in an isotropic solid. They also considered supersonic screw dislocations. In this paper an explicit expression for the drag force for a general anisotropic solid is derived. It is shown that the expression is closely related to the prelogarithmic energy factor for a steadily moving dislocation.

The plan of the paper is as follows. First the formalism of Wu is outlined. Next the fundamental solution to the third problem mentioned above is derived. The complete solution of the moving dislocation problem is constructed using the fundamental solution in Sect. 4. In Sect. 5, an explicit expression for the energy flux radiated from the moving dislocation is obtained and the drag force calculated. Special attention is paid to the anisotropy effect on the drag force. Finally, some concluding remarks are made.

\section{Formulation}

For two-dimensional deformation in which the Cartesian components of the stress $\sigma_{i j}$ and the displacement $u_{i}, i, j=1,2,3$, are independent of $x_{3}$, the equations of motion are

$\mathbf{t}_{1,1}+\mathbf{t}_{2,2}=\varrho \ddot{\mathbf{u}}$,

where $\mathbf{t}_{1}=\left(\sigma_{11}, \sigma_{21}, \sigma_{31}\right)^{\mathrm{T}}, \mathbf{t}_{2}=\left(\sigma_{12}, \sigma_{22}, \sigma_{32}\right)^{\mathrm{T}}$, $\varrho$ is the density, a subscript comma denotes partial differentiation with respect to the coordinates, and an overhead dot designates a derivative with respect to time t. The stress-strain laws are

$\mathbf{t}_{1}=\mathbf{Q} \mathbf{u}_{, 1}+\mathbf{R} \mathbf{u}_{, 2}$

$\mathbf{t}_{1}=\mathbf{R}^{\mathrm{T}} \mathbf{u}_{1,}+\mathbf{T} \mathbf{u}_{1,}$

where the matrices $\mathbf{Q}, \mathbf{R}$ and $\mathbf{T}$ are given by

$Q_{i k}=C_{i 1 k 1}, R_{i k}=C_{i 1 k 2}, T_{i k}=C_{i 2 k 2}$,

$C_{i j k s}$ being the elastic constants.

For self-similar problems, the velocity and displacement gradients may be expressed as [10]

$\dot{\mathbf{u}}\left(x_{1}, x_{2}, t\right)=-2 \operatorname{Re}\left[\sum_{k=1}^{3} f_{k}\left(\omega_{k}\right) \omega_{k} \frac{\partial \omega_{k}}{\partial x_{1}} \mathbf{a}_{k}\left(\omega_{k}\right)\right]$,
$\mathbf{u}\left(x_{1}, x_{2}, t\right)_{, 1}=2 \operatorname{Re}\left[\sum_{k=1}^{3} f_{k}\left(\omega_{k}\right) \frac{\partial \omega_{k}}{\partial x_{1}} \mathbf{a}_{k}\left(\omega_{k}\right)\right]$,
$\mathbf{u}\left(x_{1}, x_{2}, t\right)_{, 2}=2 \operatorname{Re}\left[\sum_{k=1}^{3} f_{k}\left(\omega_{k}\right) p_{k}\left(\omega_{k}\right) \frac{\partial \omega_{k}}{\partial x_{1}} \mathbf{a}_{k}\left(\omega_{k}\right)\right]$, 
and the stresses as

$\mathbf{t}_{1}\left(x_{1}, x_{2}, t\right)=2 \operatorname{Re}\left[\sum_{k=1}^{3} f_{k}\left(\omega_{k}\right) \frac{\partial \omega_{k}}{\partial x_{1}}\left(\varrho \omega_{k}^{2} \mathbf{a}_{k}-p_{k}\left(\omega_{k}\right) \mathbf{b}_{k}\left(\omega_{k}\right)\right)\right]$

$\mathbf{t}_{2}\left(x_{1}, x_{2}, t\right)=2 \operatorname{Re}\left[\sum_{k=1}^{3} f_{k}\left(\omega_{k}\right) \frac{\partial \omega_{k}}{\partial x_{1}} \mathbf{b}_{k}\left(\omega_{k}\right)\right]$

where Re stands for the real part,

$\omega_{k}=y_{1}+p_{k}\left(\omega_{k}\right) y_{2}, \quad y_{1}=x_{1} / t, \quad y_{2}=x_{2} / t$

$\frac{\partial \omega_{k}}{\partial x_{1}}=\frac{1}{t-p_{k}^{\prime}\left(\omega_{k}\right) x_{2}}$

and $p_{k}^{\prime}\left(\omega_{k}\right)$ is the derivative of $p_{k}\left(\omega_{k}\right)$ with respect to $\omega_{k}$. Note that $\frac{\partial \omega_{k}}{\partial x_{1}}$ is denoted as $\frac{1}{\Delta_{k}^{\prime}}$ in [10]. The quantities $p_{k}(\omega)$ and $\mathbf{a}_{k}(\omega)$ are, respectively, the eigenvalue and eigenvector of the following eigenvalue problem:

$\left[\mathbf{Q}+p\left(\mathbf{R}+\mathbf{R}^{\mathrm{T}}\right)+p^{2} \mathbf{T}-\varrho \omega^{2} \mathbf{I}\right] \mathbf{a}(\omega)=0$

where $\mathbf{I}$ is the identity matrix. The vector $b_{k}(\omega)$ is given by

$\mathbf{b}_{k}(\omega)=\left(\mathbf{R}^{\mathrm{T}}+p_{k}(\omega) \mathbf{T}\right) \mathbf{a}_{k}(\omega)$

The eigenvalues $p_{k}(\omega)$ are selected such that the imaginary parts are positive when they are complex.

The expressions for $\omega_{k}$ as functions of $y_{1}$ and $y_{2}$ are determined by solving Eqs. (9) and (11) simultaneously. Alternatively, the expressions for $p_{k}\left(\omega_{k}\right)$ and $\mathbf{a}_{k}(\omega)$ as functions of $y_{1}$ and $y_{2}$ may be obtained directly from the following six-dimensional eigenvalue problem [10]:

$\tilde{\mathbf{N}} \boldsymbol{\xi}=p \xi$

where

$\tilde{\mathbf{N}}=\left(\begin{array}{cc}\tilde{\mathbf{N}}_{1} & \tilde{\mathbf{N}}_{2} \\ \tilde{\mathbf{N}}_{3} & \tilde{\mathbf{N}}_{1}^{\mathrm{T}}\end{array}\right), \quad \xi=\left[\begin{array}{c}\mathbf{a} \\ \tilde{\mathbf{b}}\end{array}\right]$,

$\tilde{\mathbf{N}}_{1}=-\tilde{\mathbf{T}} \tilde{\mathbf{R}}^{\mathrm{T}}, \quad \tilde{\mathbf{N}}_{2}=\tilde{\mathbf{T}}^{-1}, \quad \tilde{\mathbf{N}}_{3}=\tilde{\mathbf{R}} \tilde{\mathbf{T}}^{-1} \tilde{\mathbf{R}}^{\mathrm{T}}-\tilde{\mathbf{Q}}$

$\tilde{\mathbf{Q}}=\mathbf{Q}-\varrho y_{1}^{2} \mathbf{I}, \quad \tilde{\mathbf{R}}=\mathbf{R}-\varrho y_{1} y_{2} \mathbf{I}, \quad \tilde{\mathbf{T}}=\mathbf{T}-\varrho y_{2}^{2} \mathbf{I}$

The vector $\mathbf{b}_{k}\left(\omega_{k}\right)$ can be obtained from $\tilde{\mathbf{b}}_{k}\left(y_{1}, y_{2}\right)$ and $\mathbf{a}_{k}\left(\omega_{k}\right)$ by

$\mathbf{b}_{k}\left(\omega_{k}\right)=\tilde{\mathbf{b}}_{k}\left(y_{1}, y_{2}\right)+\varrho \omega_{k} y_{2} \mathbf{a}_{k}\left(\omega_{k}\right)$.

The quantity $\frac{\partial \omega_{k}}{\partial x_{1}}$ may be expressed as

$\frac{\partial \omega_{k}}{\partial x_{1}}=\frac{\gamma_{k}\left(\omega_{k}\right)}{t \tilde{\gamma}\left(y_{1}, y_{2}\right)}$,

where $\tilde{\gamma}_{k}\left(y_{1}, y_{2}\right)=2 \mathbf{a}_{k}^{T}\left(\omega_{k}\right) \tilde{\mathbf{b}}_{k}\left(y_{1}, y_{2}\right)$ and $\gamma_{k}\left(\omega_{k}\right)=2 \mathbf{a}_{k}^{\mathrm{T}}\left(\omega_{k}\right) \mathbf{b}_{k}\left(\omega_{k}\right)$. Since $\tilde{\mathbf{N}}_{2}$ and $\tilde{\mathbf{N}}_{3}$ are symmetric, the left eigenvector, $\boldsymbol{\eta}$, of $\tilde{\mathbf{N}}$ defined by

$\tilde{\mathbf{N}}^{\mathrm{T}} \mathbf{\eta}=p \boldsymbol{\eta}$ 
is given by

$\boldsymbol{\eta}=\left[\begin{array}{c}\tilde{\mathbf{b}} \\ \mathbf{a}\end{array}\right]$.

The corresponding left and right eigenvectors satisfy the orthogonality relations

$\frac{1}{\tilde{\gamma}_{k}} \boldsymbol{\eta}_{j}^{\mathrm{T}} \xi_{k}=\delta_{j k}$,

where $\delta_{j k}$ is Kronecker's delta. Equation (17) implies

$\sum_{k=1}^{6} \frac{1}{\tilde{\gamma}} \boldsymbol{\xi}_{k} \mathbf{\eta}_{k}^{\mathrm{T}}=\mathbf{I}_{6}$

or

$\sum_{k=1}^{6} \frac{1}{\tilde{\gamma}_{k}} \mathbf{a}_{k} \tilde{\mathbf{b}}_{k}^{\mathrm{T}}=\mathbf{I}$

$\sum_{k=1}^{6} \frac{1}{\tilde{\gamma}_{k}} \mathbf{a}_{k} \mathbf{a}_{k}^{\mathrm{T}}=\mathbf{0}=\sum_{k=1}^{6} \frac{1}{\tilde{\tilde{\gamma}}_{k}} \tilde{\mathbf{b}}_{k} \tilde{\mathbf{b}}_{k}^{\mathrm{T}}$,

where $\mathbf{I}_{6}$ is the six by six identity matrix. From Eqs. (19) and (20), we can let [14]

$\sum_{k=1}^{3} \frac{1}{\tilde{\gamma}_{k}} \mathbf{a}_{k} \tilde{\mathbf{b}}_{k}^{\mathbf{T}}=\frac{1}{2}(\mathbf{I}-i \mathbf{S})$

$\sum_{k=1}^{3} \frac{1}{\tilde{\gamma}_{k}} \mathbf{a}_{k} \mathbf{a}_{k}^{\mathrm{T}}=-\frac{i}{2} \mathbf{H}$

$\sum_{k=1}^{3} \frac{1}{\tilde{\gamma}_{k}} \tilde{\mathbf{b}}_{k} \tilde{\mathbf{b}}_{k}^{\mathrm{T}}=\frac{i}{2} \mathbf{L}$

where $\mathbf{S}, \mathbf{H}$, and $\mathbf{L}$ are referred to as the Barnett-Lothe tensors. The Barnett-Lothe tensors are real in the subsonic regime, complex in the intersonic regime, and purely imaginary in the supersonic regime. For $y_{2}=0, \mathbf{b}_{k}\left(y_{1}\right)=\tilde{\mathbf{b}}_{k}\left(y_{1}\right)$ so that Eqs. (21) and (23) yield

$\sum_{k=1}^{3} \frac{1}{\hat{\gamma}_{k}\left(y_{1}\right)} \mathbf{a}_{k}\left(y_{1}\right) \mathbf{b}_{k}^{\mathrm{T}}\left(y_{1}\right)=\frac{1}{2}\left(\mathbf{I}-i \mathbf{S}\left(y_{1}\right)\right)$

$\sum_{k=1}^{3} \frac{1}{\hat{\gamma}_{k}\left(y_{1}\right)} \mathbf{b}_{k}\left(y_{1}\right) \mathbf{b}_{k}^{\mathrm{T}}\left(y_{1}\right)=\frac{i}{2} \mathbf{L}\left(y_{1}\right)$

For orthotropic material with the symmetry planes coinciding with the coordinate planes, explicit expressions for $\mathbf{L}\left(y_{1}\right)$ and $\mathbf{S}\left(y_{1}\right)$ have been derived by Dongye and Ting [15]. The nonzero components of $\mathbf{L}\left(y_{1}\right)$ and $\mathbf{S}\left(y_{1}\right)$ are

$\alpha_{2} \sqrt{\frac{C_{22}}{C_{66}}} L_{11}\left(y_{1}\right)=\alpha_{1} \sqrt{\frac{C_{11}}{C_{66}}} L_{22}\left(y_{1}\right)=\frac{Q}{\sqrt{Y}}$,
$L_{33}\left(y_{1}\right)=\alpha_{3} \sqrt{C_{55} C_{44}}$, 
and

$-\alpha_{1} \sqrt{\frac{C_{11}}{C_{66}}} S_{12}\left(y_{1}\right)=\alpha_{2} \sqrt{\frac{C_{22}}{C_{66}}} S_{21}\left(y_{1}\right)=\frac{\alpha_{1} \sqrt{C_{11} C_{22}}-\alpha_{2} C_{12}}{\sqrt{Y}}$,

where $C_{i j}$ is the contracted notation for the elastic constants, and

$Q\left(y_{1}\right)=\alpha_{2}\left(\alpha_{1}^{2} C_{11} C_{22}-C_{12}^{2}\right)-\left(1-\alpha_{2}^{2}\right) \alpha_{1} C_{66} \sqrt{C_{22} C_{11}}$,

$Y\left(y_{1}\right)=\left(\alpha_{1} \sqrt{C_{11} C_{22}}+\alpha_{2} C_{66}\right)^{2}-\left(C_{12}+C_{66}\right)^{2}$.

Here $\alpha_{k}$ is given by

$\alpha_{k}=\sqrt{1-\left(\frac{y_{1}}{c_{k}}\right)^{2}}$,

where

$c_{1}=\sqrt{\frac{C_{11}}{\varrho}}, \quad c_{2}=\sqrt{\frac{C_{66}}{\varrho}}, \quad c_{3}=\sqrt{\frac{C_{55}}{\varrho}}$.

For isotropic materials, Eqs. (26) and (27) can be simplified as

$\alpha_{2} L_{11}(\nu)=\alpha_{1} L_{22}(\nu)=\mu \frac{4 \alpha_{2} \alpha_{1}-\left(1+\alpha_{2}^{2}\right)^{2}}{1-\alpha_{2}^{2}}, \quad L_{33}=\mu \alpha_{2}$

and

$-\alpha_{1} S_{12}\left(y_{1}\right)=\alpha_{2} S_{21}\left(y_{1}\right)=\frac{\left(1+\alpha_{2}^{2}\right)-2 \alpha_{1} \alpha_{2}}{1-\alpha_{2}^{2}}$

\section{Fundamental solution}

In this section, the fundamental solution of a dislocation that suddenly appears at $t=0$ at the origin and moves with a constant velocity $\nu$ is derived. The dislocation motion is assumed subsonic. The continuity conditions for the traction $t_{2}^{*}$ and the jump conditions for the displacement $\mathbf{u}^{*}$ at $x_{2}=0$ are given by

$\mathbf{t}_{2}^{*}\left(x_{1}, t\right)^{+}-\mathbf{t}_{2}^{*}\left(x_{1}, t\right)^{-}=0$

$\frac{\partial}{\partial x_{1}} \mathbf{u}^{*}\left(x_{1}, t\right)^{+}-\frac{\partial}{\partial x_{1}} \mathbf{u}^{*}\left(x_{1}, t\right)^{-}=-\delta\left(x_{1}-\nu t\right) H(t) \boldsymbol{\beta}$,

where the superscripts + and - denote the limiting values as $x_{2} \rightarrow 0^{+}$and $x_{2} \rightarrow 0^{-}$, respectively, $\delta$ is the Dirac delta function, $H(t)$ the unit step function, and $\boldsymbol{\beta}$ the Burgers vector.

For $t>0$ substitution of Eqs. (8) and (5), respectively, into Eqs. (30) and (31) yields

$$
\begin{aligned}
& 2 \operatorname{Re}\left[\sum_{k=1}^{3}\left(f_{k}\left(y_{1}\right)^{+}-f_{k}\left(y_{1}\right)^{-}\right) \mathbf{b}_{k}\left(y_{1}\right)\right]=\mathbf{0}, \\
& 2 \operatorname{Re}\left[\sum_{k=1}^{3}\left(f_{k}\left(y_{1}\right)^{+}-f_{k}\left(y_{1}\right)^{-}\right) \mathbf{a}_{k}\left(y_{1}\right)\right]=-\delta\left(y_{1}-\nu\right) \boldsymbol{\beta} .
\end{aligned}
$$


Using Eqs. (17), (32) and (33), we have

$f_{k}\left(y_{1}\right)^{+}-f_{k}\left(y_{1}\right)^{-}=-\frac{\delta\left(y_{1}-\nu\right)}{\gamma_{k}\left(y_{1}\right)} \mathbf{b}_{k}^{\mathrm{T}} \boldsymbol{\beta}$.

The solution of $f_{k}\left(\omega_{k}\right)$ is readily obtained as

$f_{k}\left(\omega_{k}\right)=\frac{1}{2 \pi i\left(\omega_{k}-\nu\right) \gamma_{k}\left(\omega_{k}\right)} \mathbf{b}_{k}^{\mathrm{T}}\left(\omega_{k}\right) \boldsymbol{\beta}$

The corresponding $\dot{\mathbf{u}}^{*}$ and $\mathbf{t}_{2}^{*}$ are given by

$\dot{\mathbf{u}}^{*}\left(x_{1}, x_{2}, t ; \nu\right)=-\frac{1}{\pi t} \operatorname{Im}\left[\sum_{k=1}^{3} \frac{\omega_{k}}{\left(\omega_{k}-\nu\right) \tilde{\gamma}_{k}\left(y_{1}, y_{2}\right)} \mathbf{a}_{k}\left(\omega_{k}\right) \mathbf{b}_{k}^{\mathrm{T}}\left(\omega_{k}\right)\right] \boldsymbol{\beta}$,
$\mathbf{t}_{2}^{*}\left(x_{1}, x_{2}, t ; \nu\right)=-\frac{1}{\pi t} \operatorname{Im}\left[\sum_{k=1}^{3} \frac{1}{\left(\omega_{k}-\nu\right) \tilde{\gamma}_{k}\left(y_{1}, y_{2}\right)} \mathbf{b}_{k}\left(\omega_{k}\right) \mathbf{b}_{k}^{\mathrm{T}}\left(\omega_{k}\right)\right] \boldsymbol{\beta}$,

where Im stands for the imaginary part and Eq. (15) has been used.

Certain checks of the correctness of Eqs. (36) and (37) can be made. First, by taking $\nu=0$ and $t \rightarrow \infty, \omega_{k} \rightarrow 0$ such that $\omega_{k} t \rightarrow x_{1}+p_{k}(0) x_{2}$, Eq. (37) reduces to the static solution ([3], [16]). Second, at a fixed distance $\xi_{1}$ from the current position of the dislocation, $y_{1}=\xi_{1} / t+\nu$. As $t \rightarrow \infty, y_{1} \rightarrow \nu$ and $y_{2} \rightarrow 0$ so that $\omega_{k} \rightarrow \nu$ and $t\left(\omega_{k}-\nu\right) \rightarrow x_{1}-\nu t+p(\nu) x_{2}$, the steadystate solution [17] is recovered. Finally, near the dislocation, i.e., $\xi_{1} \rightarrow 0$, the steady-state solution prevails as anticipated. It is interesting to note that at $x_{2}=0, \omega_{k}=y_{1}$, and Eqs. (36) and (37) become

$\dot{\mathbf{u}}^{*}\left(x_{1}, 0, t ; \nu\right)=\frac{1}{1-\nu / y_{1}} \dot{\mathbf{u}}^{*}\left(x_{1}, 0, t ; 0\right)$,

$\mathbf{t}_{2}^{*}\left(x_{1}, 0, t ; \nu\right)=\frac{1}{1-\nu / y_{1}} \mathbf{t}_{2}^{*}\left(x_{1}, 0, t ; 0\right)$.

The solution for the moving dislocation is proportional to that for the stationary dislocation.

\section{Dislocation starting from rest and moving with constant velocity}

Consider a dislocation with the Burgers vector $\boldsymbol{\beta}$ starting from rest at $t=0$ and moving thereafter with constant velocity $\nu$. The initial position of the dislocation is assumed at the origin. The solution to the present problem can be obtained by the superposition of those to the following three problems:

Problem I: A static dislocation with the Burgers vector $\boldsymbol{\beta}$ at the origin.

Problem II: A stationary dislocation with Burgers vector $-\boldsymbol{\beta}$ that suddenly appears at $t=0$ at the origin.

Problem III: A moving dislocation with Burgers vector $\boldsymbol{\beta}$ that suddenly appears at $t=0$ at the origin and moving thereafter with constant velocity $\nu$.

The solution to Problem III is the fundamental solution derived in the preceding section. The solution to Problem I can be deduced from the fundamental solution by taking $\nu=0$ and $t \rightarrow \infty$ as discussed in the preceding section. The solution to Problem II can also be deduced from the fundamental solution by taking $\nu=0$. Thus $\dot{\mathbf{u}}$ can be expressed as

$\dot{\mathbf{u}}\left(x_{1}, x_{2}, t\right)=\dot{\mathbf{u}}^{(s)}\left(x_{1}, x_{2}\right)+\dot{\mathbf{u}}^{(d)}\left(x_{1}, x_{2}, t ; \nu\right)$, 
where $\dot{\mathbf{u}}^{(\mathrm{s})}$ and $\dot{\mathbf{u}}^{(d)}$ are defined as

$\dot{\mathbf{u}}^{(s)}\left(x_{1}, x_{2}\right)=\dot{\mathbf{u}}^{*}\left(x_{1}, x_{2}, \infty ; 0\right)$,

$\dot{\mathbf{u}}^{(d)}\left(x_{1}, x_{2}, t\right)=\dot{\mathbf{u}}^{*}\left(x_{1}, x_{2}, t ; \nu\right)-\dot{\mathbf{u}}^{*}\left(x_{1}, x_{2}, t ; 0\right)$

and $\dot{\mathbf{u}}^{*}\left(x_{1}, x_{2}, t ; \nu\right)$ is the fundamental solution of Eq. (36). The resulting expression for $\dot{\mathbf{u}}^{(\alpha)}$ is

$\dot{\mathbf{u}}^{(d)}\left(x_{1}, x_{2}, t\right)=-\frac{\nu}{\pi t} \operatorname{Im}\left[\sum_{k=1}^{3} \frac{1}{\left(\omega_{k}-\nu\right) \tilde{\gamma}_{k}\left(y_{1}, y_{2}\right)} \mathbf{a}_{k}\left(\omega_{k}\right) \mathbf{b}_{k}^{\mathrm{T}}\left(\omega_{k}\right)\right] \boldsymbol{\beta}$.

Similarly, $\mathrm{t}_{2}^{(d)}$ is given by

$\mathbf{t}_{2}^{(d)}\left(x_{1}, x_{2}, t\right)=-\frac{\nu}{\pi t} \operatorname{Im}\left[\sum_{k=1}^{3} \frac{1}{\omega_{k}\left(\omega_{k}-\nu\right) \tilde{\gamma}_{k}\left(y_{1}, y_{2}\right)} \mathbf{b}_{k}\left(\omega_{k}\right) \mathbf{b}_{k}^{\mathrm{T}}\left(\omega_{k}\right)\right] \boldsymbol{\beta}$.

At $x_{2}=0, \omega_{k}=y_{1}$, and Eqs. (40) and (4l) may be simplified as

$\dot{\mathbf{u}}^{(d)}\left(x_{1}, t\right)=\frac{\nu}{2 \pi\left(x_{1}-\nu t\right)} \mathbf{S}\left(y_{1}\right) \boldsymbol{\beta}$,

$\mathbf{t}_{2}^{(d)}\left(x_{1}, t\right)=\frac{\nu}{2 \pi x_{1}\left(y_{1}-\nu\right)} \mathbf{L}\left(y_{1}\right) \boldsymbol{\beta}$,

where Eqs. (24) and (25) have been substituted. It should be mentioned that in general $\mathbf{S}\left(y_{1}\right)$ and $\mathbf{L}\left(y_{1}\right)$ are full matrices so that for an edge dislocation, i.e., $\boldsymbol{\beta}=\beta_{1} \mathrm{e}, \sigma_{22}^{(d)}$ as well as $\dot{u}_{3}^{(d)}$ do not vanish at $x_{2}=0$. Thus, the boundary conditions $\sigma_{22}^{(d)}=u_{3}^{(d)}=0$ at $x_{2}=0$ assumed in [8] are valid only for materials such as orthotropic materials which possess certain symmetries to render $L_{21}=S_{31}=0$.

For isotropic materials, $\mathbf{a}_{k}\left(\omega_{k}\right)$ may be taken as

$\mathbf{a}_{1}\left(\omega_{1}\right)=\left[\begin{array}{c}1 \\ p_{1}\left(\omega_{1}\right) \\ 0\end{array}\right], \quad \mathbf{a}_{2}\left(\omega_{2}\right)=\left[\begin{array}{c}-p_{2}\left(\omega_{2}\right) \\ 1 \\ 0\end{array}\right], \quad \mathbf{a}_{3}\left(\omega_{3}\right)=\left[\begin{array}{l}0 \\ 0 \\ 1\end{array}\right]$

and $\mathbf{b}_{k}\left(\omega_{k}\right)$ as

$\mathbf{b}_{1}\left(\omega_{1}\right)=\mu\left[\begin{array}{c}2 p_{1}\left(\omega_{1}\right) \\ p_{2}\left(\omega_{1}\right)^{2}-1 \\ 0\end{array}\right], \quad \mathbf{b}_{2}\left(\omega_{2}\right)=\mu\left[\begin{array}{c}1-p_{2}\left(\omega_{2}\right)^{2} \\ 2 p_{2}\left(\omega_{2}\right) \\ 0\end{array}\right], \quad \mathbf{b}_{3}\left(\omega_{3}\right)=\mu\left[\begin{array}{c}0 \\ 0 \\ p_{2}\left(\omega_{3}\right)\end{array}\right]$,

where $p_{k}(\omega)=\sqrt{\left(\omega / c_{k}\right)^{2}-1}, k=1,2, \mu$ is the shear modulus, and $c_{1}$ and $c_{2}$ are, respectively, the $\mathrm{P}$ wave and the $\mathrm{S}$ wave speeds. The explicit expressions for $\omega_{k}$ and $\tilde{\gamma}_{k}$ are

$$
\begin{aligned}
& \omega_{k}=\frac{y_{1}+y_{2} \sqrt{\left(\frac{y}{c_{k}}\right)^{2}-1}}{1-\left(\frac{y_{2}}{c_{k}}\right)^{2}}, \quad k=1,2, \quad \omega_{3}=\omega_{2}, \\
& \hat{\gamma}_{k}=2 \mu\left(\frac{\omega_{k}}{c_{2}}\right)^{2} \sqrt{\left(\frac{y}{c_{k}}\right)^{2}-1}, \quad k=1,2, \quad \hat{\gamma}_{3}=2 \mu \sqrt{\left(\frac{y}{c_{2}}\right)^{2}-1},
\end{aligned}
$$


where $y=\sqrt{y_{1}^{2}+y_{2}^{2}}$. Equations (40) and (41) specialized for isotropic materials agree with those obtained by Markenscoff [4] for a screw dislocation and by Markenscoff and Clifton [5] for an edge dislocation.

\section{Energy radiated from moving dislocations}

The energy flux $\dot{E}$ flowing into the core of a dislocation moving along the $x_{1}$-axis can be calculated by [18]

$$
\dot{E}=\lim _{\Gamma \rightarrow 0} \int_{\Gamma}\left(\sigma_{i j} n_{j} \dot{u}_{i}+\frac{1}{2}\left(\sigma_{i j} u_{i, j}+\varrho \dot{u}_{i} \dot{u}_{i}\right) \nu n_{1}\right) d \Gamma,
$$

where $\Gamma$ is an arbitrary closed contour around the dislocation, $n_{1}$ the unit normal vector directed away from the dislocation, and the limit indicates that $\Gamma$ is shrunk onto the core of the dislocation. In particular, if $\Gamma$ is chosen to be a rectangle formed by $\xi_{1}=x_{1}-\nu t= \pm \delta_{1}$ and $x_{2}=\delta_{2}$, Eq. (44) becomes [18]

$\dot{E}=2 \lim _{\delta_{1} \rightarrow 0}\left(\lim _{\delta_{2} \rightarrow 0} \int_{-\delta_{1}}^{\delta_{1}} \mathbf{t}_{2}^{\mathrm{T}}\left(\xi_{1}, \delta_{2}, t\right) \dot{\mathbf{u}}\left(\xi_{1}, \delta_{2}, t\right) d \xi_{1}\right)$.

Near the dislocation at $x_{2}=\delta_{2} \rightarrow 0$, the particle velocity $\dot{\mathbf{u}}$ and the stress $t_{2}$ can be expanded as

$$
\begin{aligned}
& \dot{\mathbf{u}}\left(\xi_{1}, \delta_{2}, t\right)=\dot{\mathbf{u}}^{(1)}\left(\xi_{1}\right)+\dot{\mathbf{u}}^{(2)}\left(\xi_{1}, \delta_{2}\right), \\
& \mathbf{t}_{2}\left(\xi_{1}, \delta_{2}, t\right)=\mathbf{t}_{2}^{(1)}\left(\xi_{1}\right)+\mathbf{t}_{2}^{(2)}(t),
\end{aligned}
$$

where

$$
\begin{aligned}
& \dot{\mathbf{u}}^{(1)}\left(\xi_{1}\right)=\frac{\nu}{2 \pi \xi_{1}} \mathbf{S}(\nu) \boldsymbol{\beta} \\
& \dot{\mathbf{u}}^{(2)}\left(\xi_{1}, \delta_{2}\right)=\frac{\nu}{\pi} \operatorname{Re}\left[\sum_{k=1}^{3} \frac{\varepsilon_{k}}{\xi_{1}^{2}+\varepsilon_{k}^{2}} \frac{\mathbf{a}_{k}(\nu) \mathbf{b}_{k}^{\mathrm{T}}(\nu)}{\gamma_{k}(\nu, 0)}\right] \boldsymbol{\beta} \\
& \mathbf{t}_{2}^{(1)}\left(\xi_{1}\right)=\frac{1}{2 \pi \xi_{1}} \mathbf{L}(\nu) \boldsymbol{\beta} \\
& \mathbf{t}_{2}^{(2)}(t)=\frac{1}{2 \pi \nu t}\left(\nu^{2} \frac{d}{d \nu}\left(\frac{\mathbf{L}(\nu)}{\nu}\right)+\mathbf{L}(0)\right) \boldsymbol{\beta},
\end{aligned}
$$

in which $\varepsilon_{k}=\operatorname{Im}\left[p_{k}(\nu)\right] \delta_{2}$. The terms $\dot{\mathbf{u}}^{(1)}\left(\xi_{1}\right)$ and $\mathbf{t}_{2}^{(1)}\left(\xi_{1}\right)$ are the same as for a steadily moving dislocation [17]. The first and the second term in Eq. (48) are from $\mathbf{t}_{2}^{(d)}$ and $\mathbf{t}_{2}^{(s)}$, respectively. With Eqs. (46) and (47), the integrand of Eq. (45) is given by

$$
\begin{aligned}
\mathbf{t}_{2}^{\mathrm{T}}\left(\xi_{1}, \delta_{2}, t\right) \dot{\mathbf{u}}\left(\xi_{1}, \delta_{2}, t\right)= & \mathbf{t}_{2}^{(1) \mathrm{T}}\left(\xi_{1}\right) \dot{\mathbf{u}}^{(1)}\left(\xi_{1}\right)+\mathbf{t}_{2}^{(1) \mathrm{T}}\left(\xi_{1}\right) \dot{\mathbf{u}}^{(2)}\left(\xi_{1}, \delta_{2}\right) \\
& +\mathbf{t}_{2}^{(2) \mathrm{T}}(t) \dot{\mathbf{u}}^{(1)}\left(\xi_{1}\right)+\mathbf{t}_{2}^{(2) \mathrm{T}}(t) \dot{\mathbf{u}}^{(2)}\left(\xi_{1}, \delta_{2}\right)
\end{aligned}
$$

The first term vanishes as it contains $\boldsymbol{\beta}^{\mathrm{T}} \mathbf{L}(\nu) \mathbf{S}(\nu) \boldsymbol{\beta}$, and $\mathbf{L}(\nu) \mathbf{S}(\nu)$ is anti-symmetric [14]. The second and third terms are odd functions of $\xi_{1}$ and do not contribute to the integral. Thus 
only the last term is needed in evaluating Eq. (45). The result is

$\dot{E}(\nu)=\frac{1}{2 \pi t} \boldsymbol{\beta}^{\mathrm{T}}\left(\mathbf{L}^{\prime}(\nu) \nu-\mathbf{L}(\nu)+\mathbf{L}(0)\right) \boldsymbol{\beta}$

where Eq. (24) has been used. With Eq. (28) substituted, Eq. (49) recovers the result for isotropic materials derived by Clifton and Markenscoff [13]. It is interesting to note that Eq. (49) may be expressed as

$\dot{E}(\nu)=\frac{2}{t}(h(0)-h(\nu))$,

where $h$ is the prelogarithmic energy factor for a steadily moving dislocation given as [19]

$h(\nu)=\frac{1}{4 \pi} \boldsymbol{\beta}^{\mathrm{T}}\left(\mathbf{L}(\nu)-\mathbf{L}^{\prime}(\nu) \nu\right) \boldsymbol{\beta}$.

The matrix $\mathbf{L}(\nu)$ can be computed by [20]

$\mathbf{L}(\nu)=-\frac{1}{\pi} \int_{0}^{\pi} \mathbf{N}_{3}(\theta, \nu) d \theta$,

where

$\mathbf{N}_{3}(\theta, \nu)=\mathbf{R}(\theta, \nu) \mathbf{T}^{-1}(\theta, \nu) \mathbf{R}^{\mathrm{T}}(\theta, \nu)-\mathbf{Q}(\theta, \nu)$,

$\mathbf{Q}(\theta, \nu)=\mathbf{Q} \cos ^{2} \theta+\left(\mathbf{R}+\mathbf{R}^{\mathbf{T}}\right) \cos \theta \sin \theta+\mathbf{T} \sin ^{2} \theta-\varrho \nu^{2} \cos ^{2} \theta \mathbf{I}$,

$\mathbf{R}(\theta, \nu)=\mathbf{R} \cos ^{2} \theta+(\mathbf{T}-\mathbf{Q}) \cos \theta \sin \theta-\mathbf{R}^{\mathbf{T}} \sin ^{2} \theta+\varrho \nu^{2} \cos \theta \sin \theta \mathbf{I}$,

$\mathbf{T}(\theta, \nu)=\mathbf{T} \cos ^{2} \theta-\left(\mathbf{R}+\mathbf{R}^{\mathrm{T}}\right) \cos \theta \sin \theta+\mathbf{Q} \sin ^{2} \theta-\varrho \nu^{2} \sin ^{2} \mathbf{I}$.

The derivative of $\mathbf{N}_{3}(\theta, \nu)$ with respect to $\nu, \mathbf{N}_{3}^{\prime}(\theta, \nu)$, may be expressed as [21]

$\mathbf{N}_{3}^{\prime}(\theta, \nu)=2 \varrho \nu \mathbf{X}^{\mathrm{T}} \mathbf{X}, \quad \mathbf{X}=\cos \theta \mathbf{I}+\sin \theta \mathbf{T}^{-1}(\theta, \nu) \mathbf{R}^{\mathrm{T}}(\theta, \nu)$.

Equation (51) implies that $\mathbf{L}^{\prime}(0)=0$ and $\mathbf{L}^{\prime}(\nu)$ is negative-definite for $\nu>0$. Taking the derivative of Eq. (51) with respect to $\nu$ yields

$\mathbf{N}_{3}^{\prime \prime}(\theta, \nu)=2 \varrho\left(\mathbf{X}^{\mathrm{T}} \mathbf{X}+\nu \mathbf{X}^{\prime \mathrm{T}} \mathbf{X}+\nu \mathbf{X}^{\mathrm{T}} \mathbf{X}^{\prime}\right)$.

It can be shown that

$\mathbf{X}^{\prime}=2 \varrho \nu \sin ^{2} \theta \mathbf{T}^{-1}(\theta, \nu) \mathbf{X}$.

With Eq. (53) substituted, Eq. (52) becomes

$\mathbf{N}_{3}^{\prime \prime}(\theta, \nu)=2 \varrho \mathbf{X}^{\mathrm{T}}\left(\mathbf{I}+4 \varrho \nu^{2} \sin ^{2} \theta \mathbf{T}^{-1}(\theta, \nu)\right) \mathbf{X}$,

where the fact that $\mathbf{T}(\theta, \nu)$ is symmetric has been used. Since $\mathbf{T}(\theta, \nu)$ is positive-definite [14], Eq. (54) implies that $\mathbf{L}^{\prime \prime}(\nu)$ is also negative-definite. From Eq. (49),

$\dot{E}^{\prime}(\nu)=\frac{\nu}{2 \pi t} \boldsymbol{\beta}^{\mathrm{T}} \mathbf{L}^{\prime \prime}(\nu) \boldsymbol{\beta}$,

so that $\dot{E}^{\prime}(\nu)<0$ for $\nu \neq 0$. Since $\dot{E}(0)=0$, it follows that $\dot{E}(\nu)<0$ for $\nu \neq 0$. Thus a positive energy flux is radiated outward from the moving dislocation. 

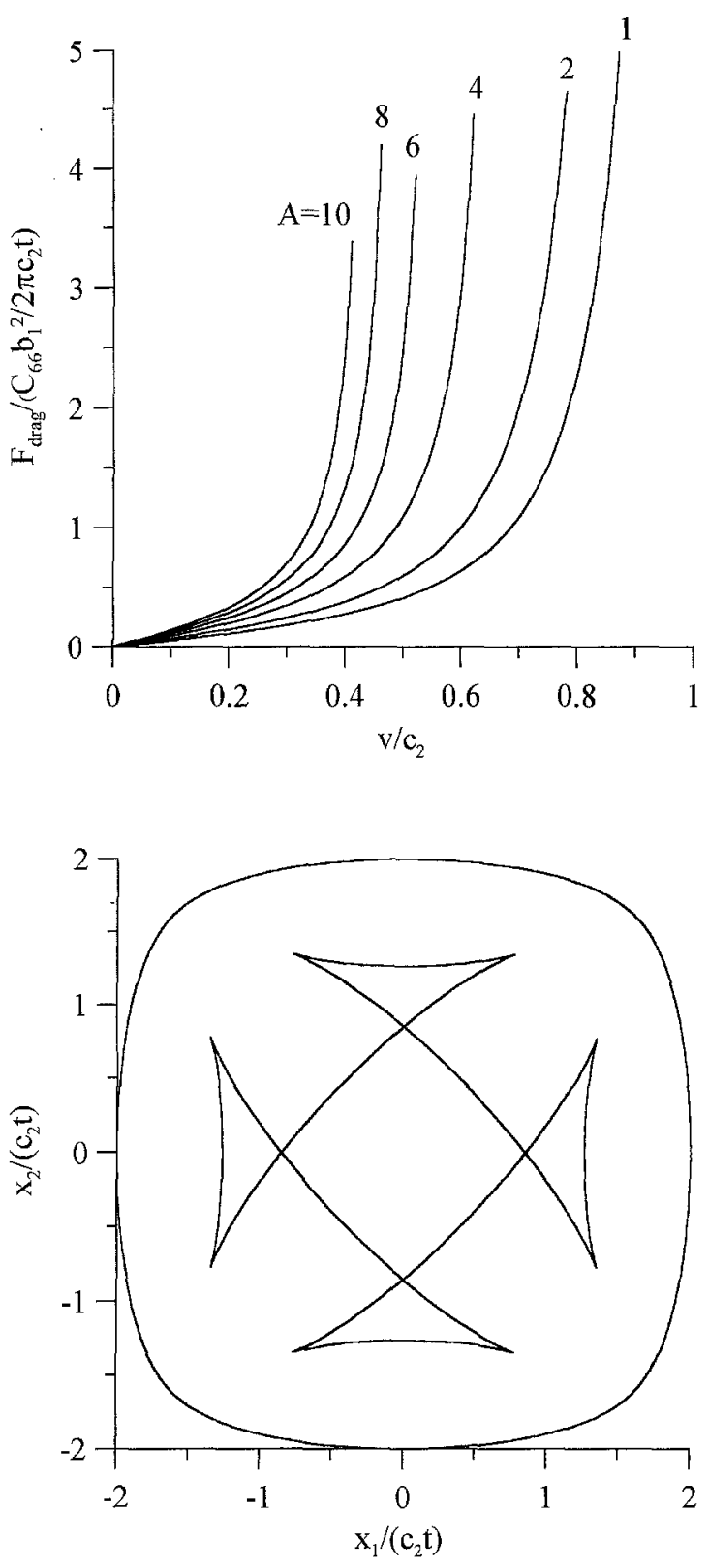

Fig. 1. The dependence of the drag force on the dislocation velocity for $A=\frac{2 C_{66}}{C_{11}-C_{12}}$ $=1,2,4,6,8,10, B=\frac{C_{12}}{2 C_{66}}=1$

Fig. 2. Typical wave front curves for cubic materials with $A=\frac{2 C_{66}}{C_{11}-C_{12}}>1.3$ and $B=\frac{C_{12}}{2 C_{66}}=1$

The drag force required for the elastic fields associated with the dislocation motion to have neither an energy source nor an energy sink at the dislocation is given by [13]

$F_{\text {drag }}=-\frac{\dot{E}}{\nu}$.

To assess the anisotropy effect on the drag force, consider an edge dislocation moving in a cubic material with the elastic constants characterized by the following two dimensionless parameters $A$ and $B$ :

$A=\frac{2 C_{66}}{C_{11}-C_{12}}, \quad B=\frac{C_{12}}{2 C_{66}}$, 
where $A>0$ and $B>-1 /(3 A)$. The material with $A=1$ corresponds to the isotropic material. The Burgers vector $\boldsymbol{\beta}$ is taken as $\boldsymbol{\beta}=b_{1} \mathbf{e}_{1}, \mathbf{e}_{1}$ being the unit vector in the $x_{1}$-direction. Figure 1 shows the dependence of the drag force on the dislocation velocity for $A=1,2,4,6,8,10$ and $B=1$. The curve for $A=1$ is the same as that obtained by Clifton and Markenscoff [13] for the isotropic material with the Poisson ratio $\nu=1 / 3$. From Eq. (49), for small dislocation velocities, the drag force can be approximated as

$F_{\text {drag }} \approx-\frac{L_{11}^{\prime \prime}(0) b_{1}^{2}}{4 \pi t} \nu$

and is linearly proportional to $\nu$. The drag force becomes infinite as a limiting speed is approached. For $A=1$, the limiting speed is the shear wave speed $c_{2}$. The limiting speeds vary from $0.90 c_{2}$ for $A=2$ to $0.44 c_{2}$ for $A=10$. To see the physical meaning of the limiting speeds, typical wave front curves for the materials with $A>1.3$ are plotted in Fig. 2 . The outer curve is the qL (quasi-Longitudinal) wave front and the inner is the qS (quasi-Shear) wave front. The $\mathrm{qS}$ wave front has four cuspidal triangles, two centered on the $x_{1}$-axis and two on the $x_{2}$-axis. The triangular regions are lacunas where there is no disturbance [22]. Thus, along the $x_{1}$-direction, in addition to the fast shear wave travelling with $c_{2}$, there is another slower shear wave. The limiting speed is actually the slower shear wave speed, which is determined by $Y(c)=0$.

\section{Conclusions}

A closed form solution to the transient problem of a dislocation starting from rest and moving with constant subsonic velocity has been presented. The materials considered are of general anisotropy and the dislocations with general Burgers vectors. The elastic fields of the moving dislocation are used to derive the energy flux radiated from the dislocation. It is shown that the energy flux may be expressed in terms of the energy factor for a steadily moving dislocation.

\section{Acknowledgement}

The research was supported by the National Science Council of Taiwan under grant No. NSC 90-2212-E$002-155$.

\section{References}

[1] Weertman, J., Weertman, J. R.: Dislocations in solids, vol. 3 (Nabarro, F. R. N., ed.). Amsterdam: North-Holland 1983.

[2] Indenbom, V. L., Lothe, J.: Elastic strain fields and dislocation mobility. Amsterdam: North-Holland 1992.

[3] Eshelby, J. D., Read, W. T., Shockley, W: Anisotropic elasticity with applications to dislocation theory. Acta Metall. 1, $251-259$ (1953).

[4] Markenscoff. X.: The transient motion of a nonuniformly moving dislocation. J. Elast. 10, 193-201 (1980).

[5] Markenscoff, X., Clifton, R. J.: The nonuniformly moving edge dislocations. J. Mech. Phys. Solids 29, 253-262 (1981). 
[6] Markenscoff, X., Ni, L.: Nonuniform motion of an edge dislocation in an anisotropic solid I. Q. Appl. Math. 41, 475-494 (1984).

[7] Markenscoff, X., Ni, L.: Nonuniform motion of an edge dislocation in an anisotropic solid II. Q. Appl. Math. 42, 425-432 (1985).

[8] Markenscoff, X.: The transient motion of a dislocation in a solid of general anisotropy. Wave Motion 9, 191 - 199 (1987).

[9] Cagniard, L.: Reflection and refraction of seismic waves (translated and revised by Flinn, E. A. and Dix, C. H.). New York: McGraw-Hill 1962.

[10] Wu, K.-C.: Extension of Stroh's formalism to self-similar problems in two-dimensional elastodynamics. Proc. Roy. Soc. London A456, 869 -890 (2000).

[11] Hirth, J. P., Lothe, J.: Theory of dislocations. New York: McGraw-Hill 1968.

[12] Eshelby, J. D.: The equation of motion of a dislocation. Phys. Rev. 90, 248-255 (1953).

[13] Clifton, R. J., Markenscoff, X.: Elastic precursor decay and radiation from nonuniformly moving dislocations. J. Mech. Phys. Solids 29, 227-251 (1981).

[14] Ting, T. C. T.: Anisotropic elasticity- theory and application. New York: Oxford University Press 1996.

[15] Dongye, C., Ting, T. C. T.: Explicit expressions of Barnett-Lothe tensors and their associated tensors for orthotropic materials. Q. Appl. Math. 47, 723-734 (1989).

[16] Stroh, A. N.: Dislocations and cracks in anisotropic elasticity. Phil. Mag. 3, 625-646 (1958).

[17] Stroh, A. N.: Steady state problems in anisotropic elasticity. J. Math. Phys. 41, 77-103(1962).

[18] Freund, L. B.: Energy flux into the tip of an extending crack in an elastic solid. J. Elast. 2, 341 - 349 (1972).

[19] Beltz, R. J., Davis, T. L., Malen, K.: Some unifying relations for moving dislocations. Phys. Stat. Sol. 26, 621-637 (1968).

[20] Barnett, D. M., Lothe, J.: Synthesis of the sextic and the integral formalism for dislocations, Green's function and surface waves in anisotropic elastic solids. Phys. Norv. 7, 13-19 (1973).

[21] Chadwick, P., Jarvis, D. A.: Surface waves in a pre-stresses elastic body. Proc. Roy. Soc. London A366, 507-536 (1979).

[22] Payton, R. G.: Elastic wave propagation in transversely isotropic media. The Hague: Martinus Nijhoff 1983.

Author's address: K.-C. Wu, Institute of Applied Mechanics, National Taiwan University, Taipei 106, Taiwan (E-mail: wukc@spring.iam.ntu.edu.tw) 